\title{
MODIFICACIÓN DEL AJUSTE DE AHNERT PARA CALCULAR PERIODOS DE RETORNO DE PRECIPITACIONES EXTREMAS EN AMBIENTES DE ELEVADA VARIABILIDAD PLUVIAL. APLICACIÓN A LA COMUNIDAD VALENCIANA
}

\author{
Martín de Luis (1), José Carlos González Hidalgo (2), \\ Josep Raventós (1) y Juan Rafael Sánchez (1) \\ (1) Dpto. de Ecología, Universidad de Alicante \\ (2) Dpto. de Geografía, Universidad de Zaragoza
}

\begin{abstract}
Resumen: Se presenta una modificación al ajuste semilogarítmico de Ahnert (1986) para el cálculo de los períodos de retorno de sucesos extremos y se comparan sus resultados con los obtenidos por el método de Gumbel. El análisis espacial de los residuos de ambas funciones en 96 observatorios de la Comunidad Valenciana sugiere que, en ambientes de extrema irregularidad pluvial como los de clima mediterráneo, la modificación propuesta en este trabajo a la función de Ahnert puede ser más precisa en el reconocimiento de los sucesos extremos.
\end{abstract}

Palabras Clave: Precipitaciones extremas. Período de retorno. Ajuste de Gumbel. Ajuste de Ahnert. Comunidad Valenciana

\begin{abstract}
A new version of Ahnert model's (1986) to calculate recurrence intervals of extreme events is presented, and the results are compared with Gumbel approach. The residual analysis of both function applied to 96 climatic stations in Comunidad Valenciana ( $E$ of Spain) suggest that in environments of extreme rainfall variability, as those under Mediterranean climate, the Ahnert modification approach can be more acute.
\end{abstract}

Key Words: Rainfall extreme events. Return periodo. Gumbel fit. Ahnert fit. Comunidad Valenciana

\section{Introducción}

En el contexto del "Cambio Global", las previsiones sobre la evolución de las precipitaciones en las áreas de clima mediterráneo son muy diversas. Tales predicciones 
varían según los modelos entre incrementos anuales del 15\% por el aumento de la humedad de la atmósfera, hasta descensos superiores al $21 \%$ originados por el supuesto incremento del albedo generador a su vez del descenso de la actividad convectiva (ver revisiones de Rowntree, 1988; Mitchell, 1989; Rowntree et al., 1993; Houghton et al., 1996).

En el conjunto de la Península Ibérica y en especial para la franja mediterránea española, numerosos trabajos han caracterizado la irregularidad de las precipitaciones (Laita y Grimalt, 1997; Llasat y Puigcerver, 1997; Goodess y Palutikof, 1998; MartínVide y Gómez, 1999), y sus tendencias (Rodríguez-Puebla et al., 1998; Esteban-Parra et al., 1998; Romero et al., 1998; De Luís 2000). Pero a pesar de que la variabilidad pluvial es un fenómeno "normal" en estos ambientes, su comportamiento ha sido calificado de excepcional en los últimos años, particularmente en la comunidad Valenciana (Quereda, 1994). Esta situación anormal pareciera estar producida por el aumento de la frecuencia de fenómenos extremos.

Por definición, cualquier suceso con valor alejado de la media ocurre menos veces que aquellos próximos al valor central. Entre ellos, los menos probables son denominados estadísticamente eventos o sucesos extremos. Generalmente en climatología reciben este calificativo aquellos eventos que superan un determinado umbral (Wigley, 1985). Sin embargo, lo que climáticamente puede denominarse extremo en una región puede no serlo en otra aunque, en ambas, tanto la naturaleza como la sociedad estén más adaptadas a los valores promedio antes que a los sucesos extraordinarios. En consecuencia, el impacto ecológico y socioeconómico del clima es sentido principalmente por los sucesos extremos los cuales pueden ser potencialmente muy destructivos.

El estudio de los eventos extremos, tanto la identificación de su frecuencia como de su magnitud, es un aspecto tratado ampliamente desde diferentes disciplinas y con diferentes objetivos en las ciencias ambientales (Chow, 1951, 1956; Benjamin, 1970; Reich, 1970, 1973; Al-Mashidani et al., 1978; Ahnert, 1986; De Ploey et al., 1991; Lewis, 1992). Particularmente en la climatología el análisis se ha centrado en el estudio de los sucesos de precipitación extrema diaria (Hershfield, 1961, 1965, 1979; Goyal y Kathuria, 1984).

Recientes estudios han puesto de manifiesto el posible cambio de los sucesos extremos de las precipitaciones en diversas partes del mundo y su posible incidencia en la variabilidad interanual de las precipitaciones y sus tendencias (Iwashima y Yamamoto, 1993; Karl et al., 1995; Yu y Neil, 1993; Suppiah y Hennessy, 1998). Sin embargo, existen notables discrepancias en la definición objetiva de lo que podrían considerarse eventos extremos, llamados generalmente torrenciales, lo cual dificulta la comparación de los citados trabajos y sus resultados. Para unos autores (Karl et al., 1995; Yu y Neil, 1993), un evento extremo debe superar un umbral (50,8 y $40 \mathrm{~mm}$ dia $^{-1}$ respectivamente), mientras que otros investigadores (Iwashima y Yamamoto, 
1993; Suppiah y Hennessy, 1998; De Luís et al., 1998), en vez de considerar umbrales volumétricos, consideran eventos torrenciales los correspondientes a los eventos máximos, segundos o terceros, en su caso, de cada año o serie de datos. Este último criterio ha sido el elegido en el presente estudio.

\section{Materiales y método}

\subsection{Los métodos de cálculo de sucesos extremos}

Uno de los aspectos más debatidos en el análisis de los sucesos extraordinarios es la adecuación de los métodos empleados, sobre todo porque de su aplicación surgirán predicciones que pueden definir actuaciones en el futuro.

Existen diversos métodos de análisis recomendados (Llamas et al., 1987; Slimani y Lebel., 1987; Lewis, 1992; Arnbjergnielsen et al., 1994; Escalante-Sandoval, 1998; Chu y Wang, 1998), cuyas principales diferencias estriban en el tipo de serie de datos a emplear, bien las series de los máximos anuales (Lana et al., 1995; Yu y Cheng, 1998), o las llamadas series parciales que incluyen valores de las diferentes series anuales a partir de umbrales determinados (Ahnert, 1986; De Ploey et al., 1991; Dupuis, 1997; Madsen et al., 1998; revisiones globales se encuentran en Sevruk y Geigr, 1981, y WMO, 1986)

Una segunda dificultad radica en la elección del tipo de distribución teórica a la que aproximar los registros empíricos. Así se sugieren las distribuciones de Gumbel I, III, Fisher y Tipett tipo II, distribuciones Beta, Pearson III, Log-Pearson III, Normal, Log-Normal, Log-Normal II, Log-Normal III, Gamma II, Gamma III, Weibull, etc.

Sin embargo, y pese a la gran variedad de métodos existentes, el método más empleado se basa en uno de los modelos generales de Jenkinson asumiendo una curvatura cero en su parámetro asintótico. Bajo esta premisa, la distribución se aproxima a una distribución de Gumbel tipo I (Gumbel, 1958) de acuerdo con las siguientes consideraciones:

Sea $P_{1}, \ldots, P_{\mathrm{n}}$ una secuencia temporal aleatoria de variables y $P_{\max }$ otra variable aleatoria definida como:

$$
P_{\max }=\max \left(P_{1}, \ldots, P_{n}\right)
$$

Asumiendo que $P_{i}(i=1, \ldots, n)$ son independientes y están distribuidos según la función

$$
F(p)=\operatorname{Prob}\left(P_{i}<p\right),(i=1, \ldots, n)
$$


entonces, la función de distribución de los máximos $P_{\max }$ queda representada por:

$$
\operatorname{Prob}\left(P_{\max }<p\right)=F^{n}(p)
$$

Si $n$ y $p$ son lo suficientemente grandes, la distribución de $P_{\max }$ queda determinada por la pendiente de la cola derecha de $F(p)$, a la que nos podemos aproximar por una distribución exponencial. Para valores elevados de $\mathrm{p}$ :

$$
\operatorname{Prob}\left(P_{\max }\right) \approx G(p)=\exp \{-\exp [-\alpha(p-u)]\}
$$

que no es más que una distribución de Gumbel tipo I con parámetros $u$ y $\alpha$.

A partir de los valores extremos empíricos, se pueden calcular su media ( $m p)$ y su desviación estándar $(\sigma p)$, que permitirán conocer los parámetros de la ecuación según las expresiones:

$$
\begin{gathered}
m p \approx u+\beta / \alpha \\
\sigma p \approx \delta / \alpha
\end{gathered}
$$

en las cuales $\beta$ y $\delta$ son coeficientes dependientes del número de valores de las series empíricas (Gumbel, 1958). Finalmente, la probabilidad de superar un valor de precipitación dado $(p)$, queda expresado como:

$$
\operatorname{Prob}\left(P_{\max }>p\right)=1-G(p)
$$

y el correspondiente periodo de retorno para un determinado valor de precipitación será:

$$
T=1 /(1-G(p))
$$

\subsection{El ajuste alternativo de Ahnert}

Como alternativa a los modelos propuestos (difíciles de aplicar hasta la generalización de los ordenadores) Ahnert (1986) ha sugerido un nuevo método empírico caracterizado por su sencillez, la facilidad para interpretar sus parámetros en términos de magnitud y frecuencia, y la posibilidad de emplear series parciales, de modo que permite integrar en el análisis los segundos, terceros etc. máximos anuales que en algunos años pueden ser superiores a los máximos de otros años.

La aproximación de Ahnert se diferencia de la aproximación clásica de Gumbel (I) en dos aspectos fundamentales (Ahnert, 1986). En primer lugar en la naturaleza de 
las propias series analizadas (series parciales) y en segundo lugar en la función de distribución empleada. En el caso de las precipitaciones, el valor esperado para un determinado retorno se calcularía, según notación de De Ploey et al., (1991)

$$
P_{24}=P_{1}+P_{0} \log _{10}(T)
$$

con $P_{24}$ el volumen de lluvia esperado en 24 horas, $P_{1}$ y $P_{0}$ los parámetros de ajuste y $T_{i}$ el valor de retorno empírico en años, obtenido según:

$$
T_{i}=n+1 / \text { rango }
$$

donde $n$ representará la longitud total de la serie (en años).

La interpretación de la función es sencilla. Convirtiendo a logaritmos los valores de retorno elegidos en el segundo término de la función, el valor de $P_{1}$ representa el retorno de 1 año, $P_{1}+P_{0}$ es el valor de retorno de 10 años; y $P_{1}+2 P_{0}$ el retorno de 100 años. De este modo los valores $P_{1}$ y $P_{0}$ son una aproximación a la magnitud y frecuencia de los eventos de cada localidad. De Ploey et al. (1991) han demostrado además que el valor del coeficiente de la ecuación $P_{0}$ representa la magnitud del día de lluvia dominante.

\subsection{El problema de los residuos y propuesta de un nuevo ajuste empírico}

Los dos ajustes reseñados, sin embargo, presentan el mismo problema de anormalidad de sus residuos (De Luís, 2000), por lo que al objeto de evitar tal situación en la distribución de éstos se propone que la función de ajuste semilogarítmica sugerida por Ahnert se sustituya por una función doble logarítmica, según:

$$
\log P_{24}=P_{1}+P_{0} \log _{10}\left(T_{i}\right)
$$

Además, dado que el objeto de estos ajustes es la caracterización en términos de magnitud-frecuencia de las precipitaciones extremas, y tras diversas calibraciones, en el presente estudio se sugiere que las series parciales sean las constituidas por los 10 eventos de precipitación máxima de cada una de las localidades para el conjunto de la serie de datos disponibles, con independencia de que procedan del mismo año o no. La justificación es que la longitud de los registros empíricos de las series originales del presente estudio varian entre los 30 y los 41 años, los cuales abarcarian desde el retorno de 4 años (10/40) hasta el retorno de 40 (1/40) años. La figura 1 muestra la solución gráfica de los tres métodos comentados en la ciudad de Alicante, que permite observar la mejora substancial que supone emplear la función de Ahnert modificada tanto respecto a su formula original como respecto al ajuste de Gumbel. 


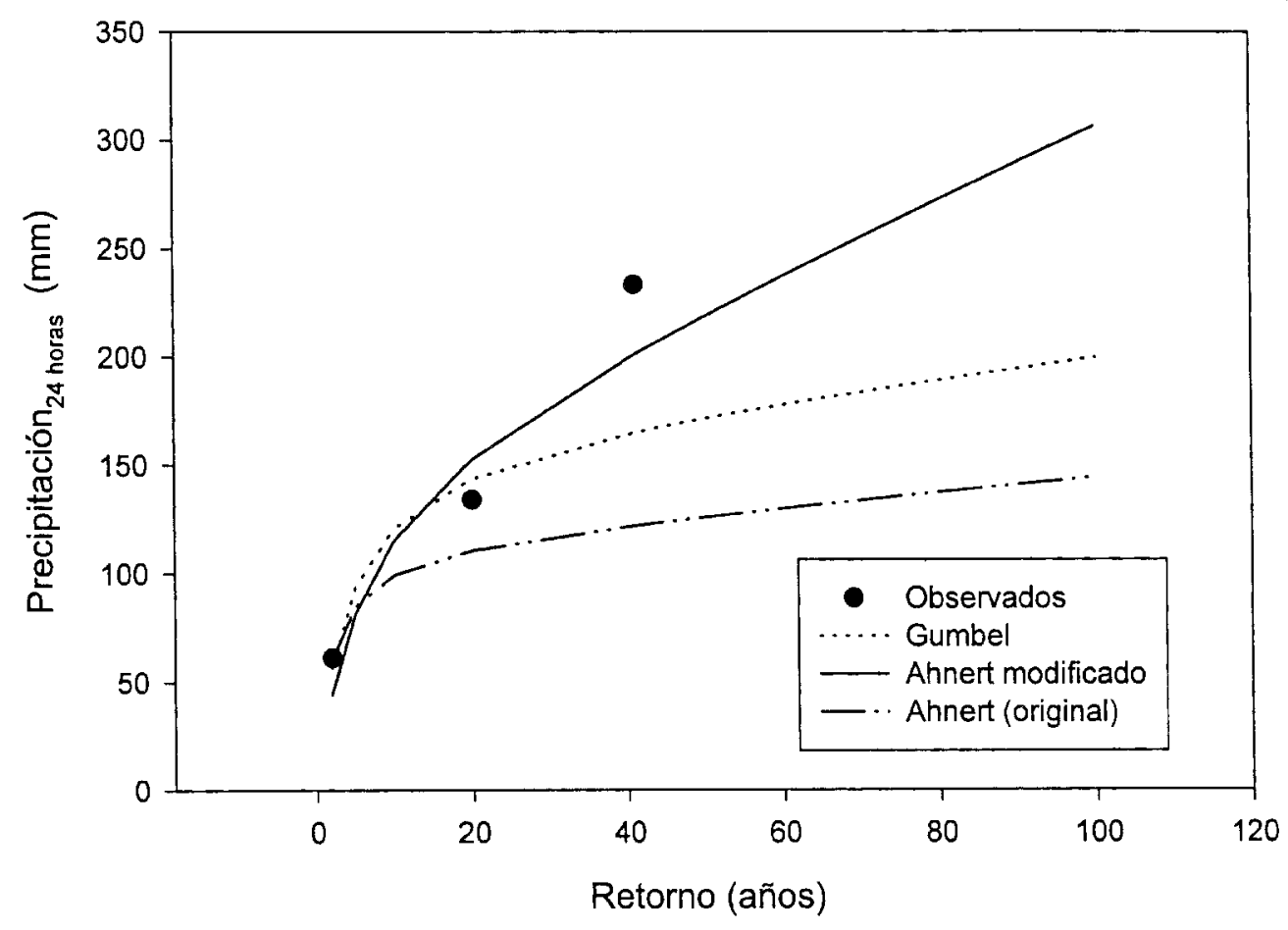

Figura 1. Observatorio de Alicante. Retorno de los valores observados ( 2 años, 20 años, 40 años) y ajustes de Gumbel, semilogarítmico de Anhert y Anhert modificado

\subsection{Aplicación y desarrollo}

En el presente estudio se ha empleado la base de datos original del Atlas Climatic de la Comunitat Valenciana (Pérez-Cueva, 1994), compuesta de más de 200 estaciones meteorológicas de las que se seleccionaron todas aquellas que contenían al menos el período normal WMO 1961-1990 al objeto de que los resultados fuesen homologables según las normas establecidas.

El total de observatorios empleados ha sido de 96, en cuya selección se tuvo en cuenta la duración de los registros y su homogeneidad. El grado de cubrimiento sobre el territorio es aceptable ( 96 observatorios para $20.000 \mathrm{~km}^{2}$ ), salvo el interior de la zona norte de la provincia de Castellón donde los observatorios son escasos.

El dato original de cada observatorio es la precipitación diaria caída durante el citado período y se refiere a 24 horas. Este hecho supone una imprecisión evidente 
que dificulta su relación con fenómenos asociados a dicha torrencialidad (erosión, avenidas, etc.). No obstante los organismos meteorológicos nacionales asumen tradicionalmente que la distribución de la precipitación diaria en la zona de estudio oscila entre las 2 y 6 horas.

En cada observatorio se calcularon los valores de retorno de lluvia diaria mediante los métodos de Gumbel, Ahnert y la modificación sugerida en este estudio al segundo de ellos. Finalmente, para calibrar su fiabilidad, en cada una de las 97 localidades se estudiaron los residuos generados por las funciones, se compararon los resultados entre las funciones y, a su vez, se contrastaron con los registros reales observados. El análisis cartográfico ha permitido establecer algunas consideraciones generales que se proponen como conclusiones provisionales.

\section{Resultados}

\subsection{Precipitaciones extremas: volúmenes máximos diarios probables}

En la Comunidad Valenciana cualquiera de las aproximaciones estadísticas empleadas (figura 2) predice unos elevados valores de precipitación cada dos años en las comarcas situadas en las proximidades del Cap de Sant Antoni. En este sector los eventos de lluvia superiores a los $100 \mathrm{~mm}$ diarios se muestran extremadamente recurrentes, y se superan los $140 \mathrm{~mm}$ cada dos años en algunos sectores. En el resto de la Comunidad se detecta un fuerte gradiente costa-interior en el reparto espacial de las cantidades de precipitación máxima esperada para este intervalo de tiempo.

Para periodos de recurrencia intermedios, 20 años, los volúmenes máximos esperados en 24 horas superan los $100 \mathrm{~mm}$ en prácticamente todo el territorio valenciano (Figura 3). De nuevo son los sectores costeros donde aparecen los valores más elevados, en torno a $140 \mathrm{~mm}$, y donde se alcanzan los máximos diarios que superan los $250 \mathrm{~mm}$ en las proximidades de Cap de Sant Antoni. El interior de la Comunidad y su extremo sur presentan valores inferiores, aunque se llega a precipitaciones en torno a los $70-80 \mathrm{~mm}$ en 24 horas.

Los valores de precipitación diaria esperados en el período de retorno de 40 años manifiestan el acusado gradiente costa-interior ya señalado (Figura 4), pero si bien en los sectores interiores de la Comunidad la magnitud de los volúmenes esperados parece haber alcanzado ya su valor absoluto (en torno a los $100 \mathrm{~mm}$ diarios, valor semejante al retorno de 20 años), en los sectores costeros la magnitud esperada de estos eventos aumenta. En todo el sector costero central los valores esperados superan con claridad los $200 \mathrm{~mm}$ y se alcanzan incluso precipitaciones superiores a los $300 \mathrm{~mm}$. 


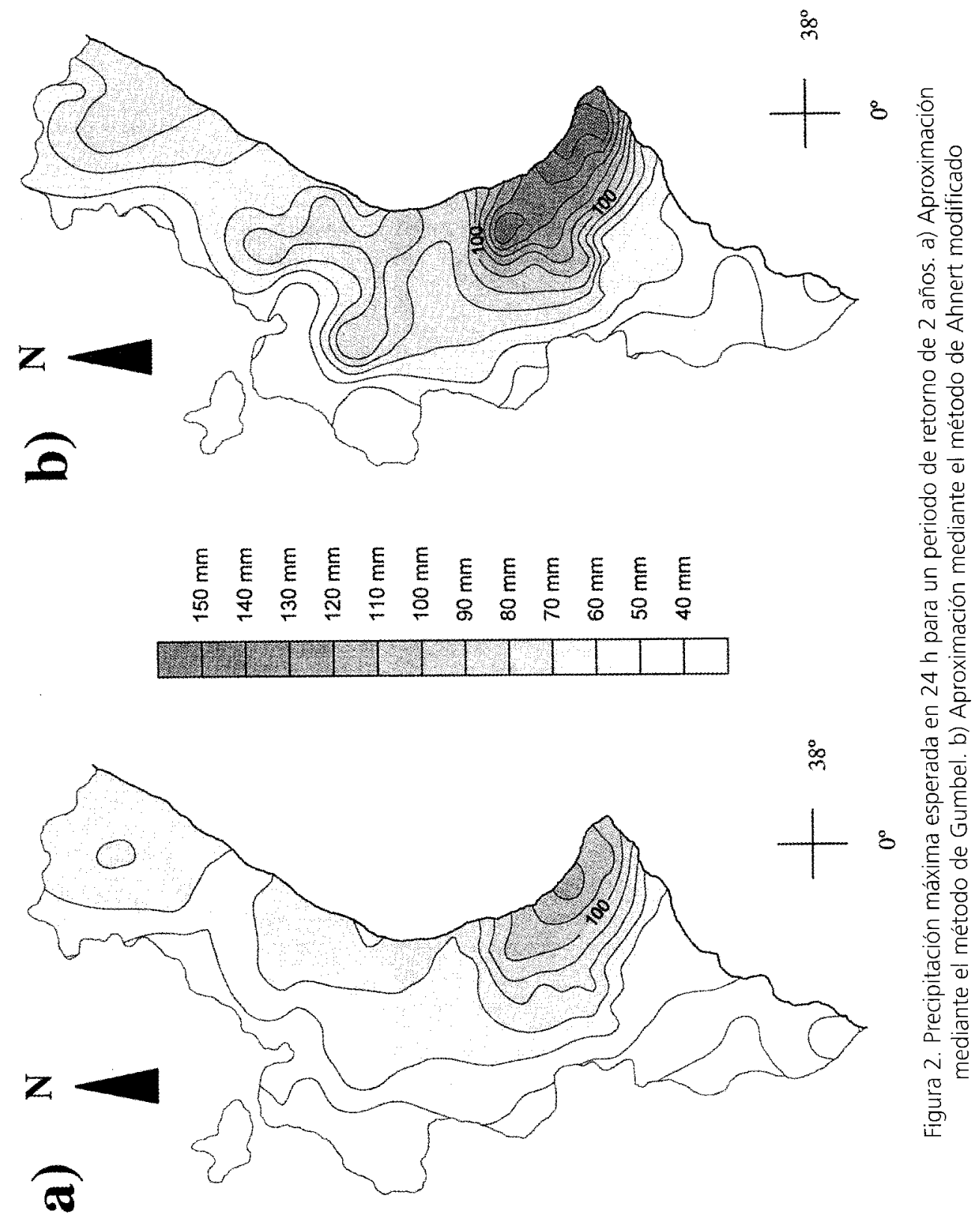




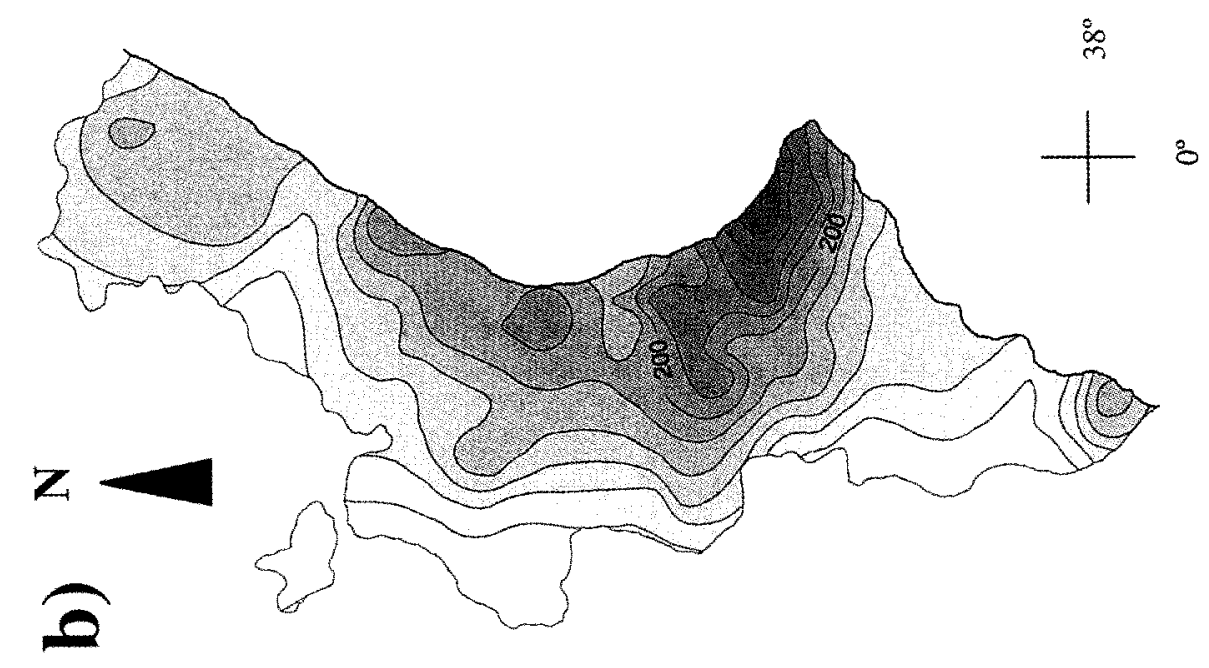

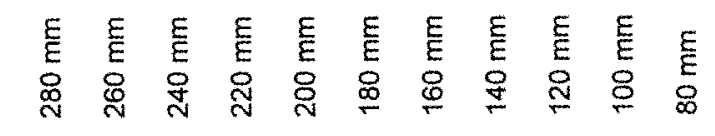
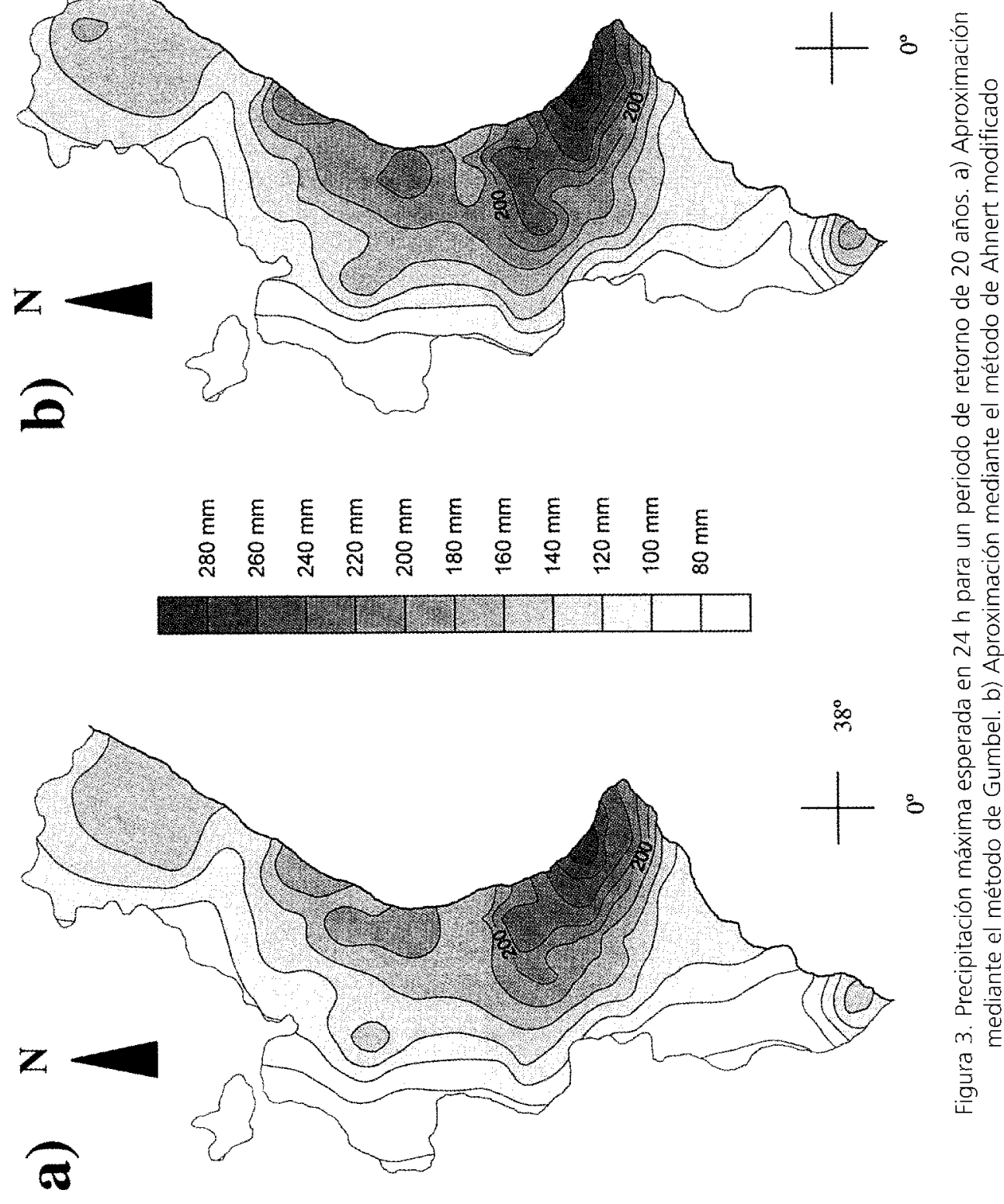

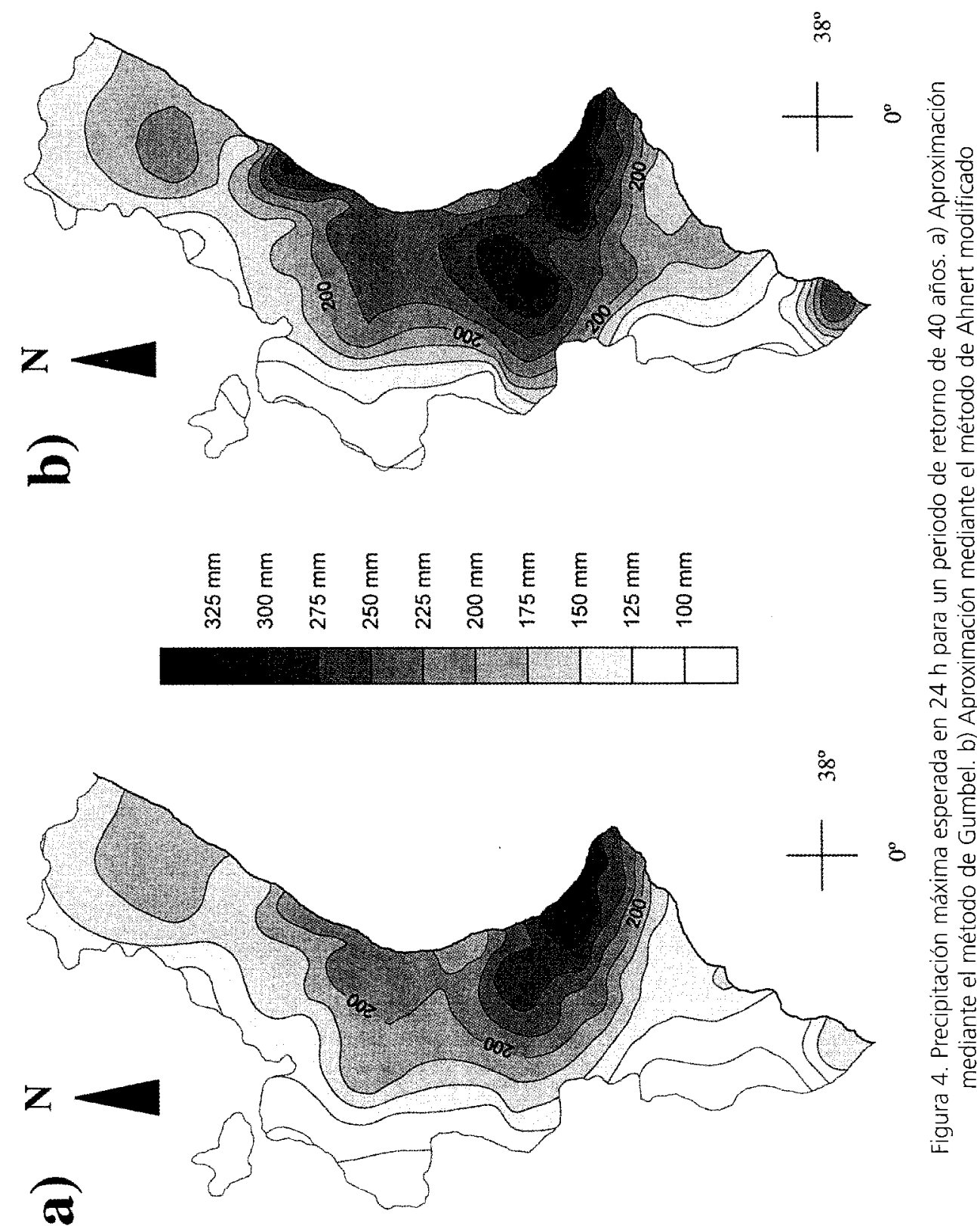

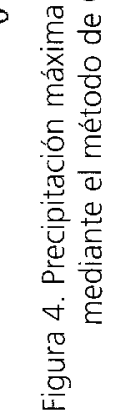




\subsection{Aspectos críticos y comparación de métodos}

Como cabe esperar por el elevado número de datos utilizados y por la propia naturaleza estadística de los métodos empleados, ambos tipos de ajustes (Gumbel y Ahnert) obtienen valores elevados de significación estadística en todas y cada una de las localidades estudiadas.

Sin embargo, para valorar la bondad de un ajuste es imprescindible considerar la normalidad de los residuos generados. Este hecho es especialmente importante si, como es el caso, el objeto del trabajo es el de utilizar un método en particular para realizar previsiones futuras.

La cartografía de los residuos ha sido la técnica empleada para contrastar ambos tipos de ajustes (Gumbel y modificación de Ahnert), analizando los residuos generados en cada caso en tres puntos concretos del dominio de la función $(2,20$ y 40 años) y en términos de porcentaje entre el valor predicho y el observado en la serie histórica.

En la Figura 5 se presenta la distribución espacial de los residuos de las previsiones efectuadas por las funciones de Gumbel y la modificación de Ahnert propuesta, respecto a los valores observados en el retorno de 2 años. Dos aspectos llaman principalmente la atención: la notable diferencia entre ambas aproximaciones se produce tanto por su diferente distribución espacial como por las diferencias en cuanto a la magnitud de las anomalías.

En general, la aproximación de Gumbel (Figura 5a) produce en este retorno una subestimación generalizada de las magnitudes de lluvia, que en gran parte del territorio valenciano superan el $15 \%$ y en la práctica totalidad el $5 \%$.

Por el contrario, las previsiones a partir del método modificado de Ahnert (Figura 5b) parecen mostrarse mucho más precisas para este retorno. Pese a la existencia de discrepancias locales en ningún caso las anomalías son extensas en el espacio y la mayor parte del territorio está afectado por valores de desviación en el intervalo $\pm 5 \%$.

En el periodo de retorno intermedio (20 años) ambos métodos parecen tener un comportamiento muy similar (Figura $6 \mathrm{a}$ y b). En ambos casos las discrepancias tienen un patrón de distribución espacial muy semejante y las desviaciones están localizadas preferentemente en el interior de la Comunidad Valenciana. Además los valores predichos por ambas funciones son también similares y la diferencia entre magnitudes predichas y observadas se localiza en el intervalo $\pm 5 \%$ en buena parte del territorio con independencia de la función empleada.

En el retorno de 40 años se vuelven a observar grandes diferencias entre ambos métodos (Figura 7 a y b). De nuevo las predicciones generadas a partir del método 


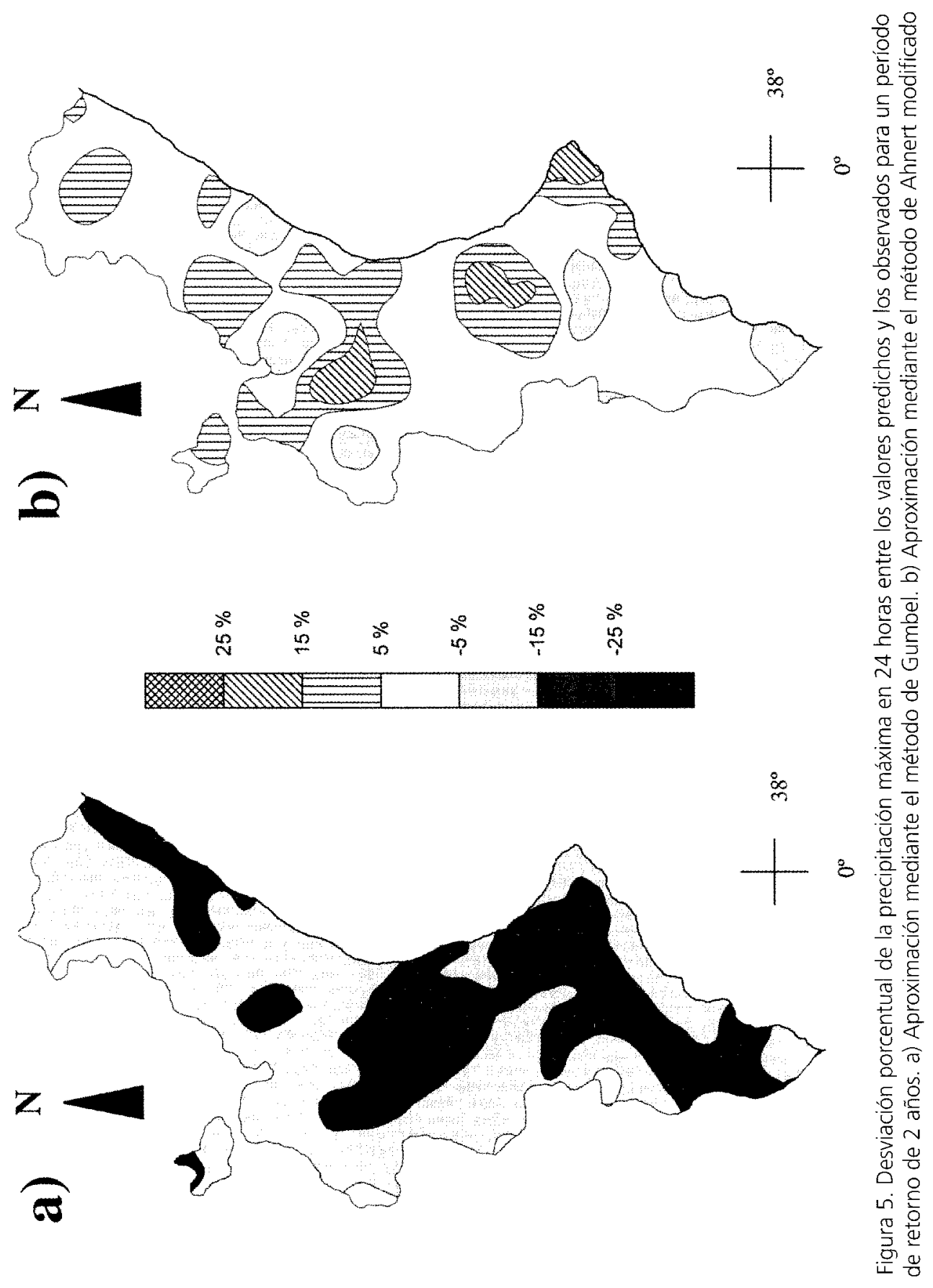




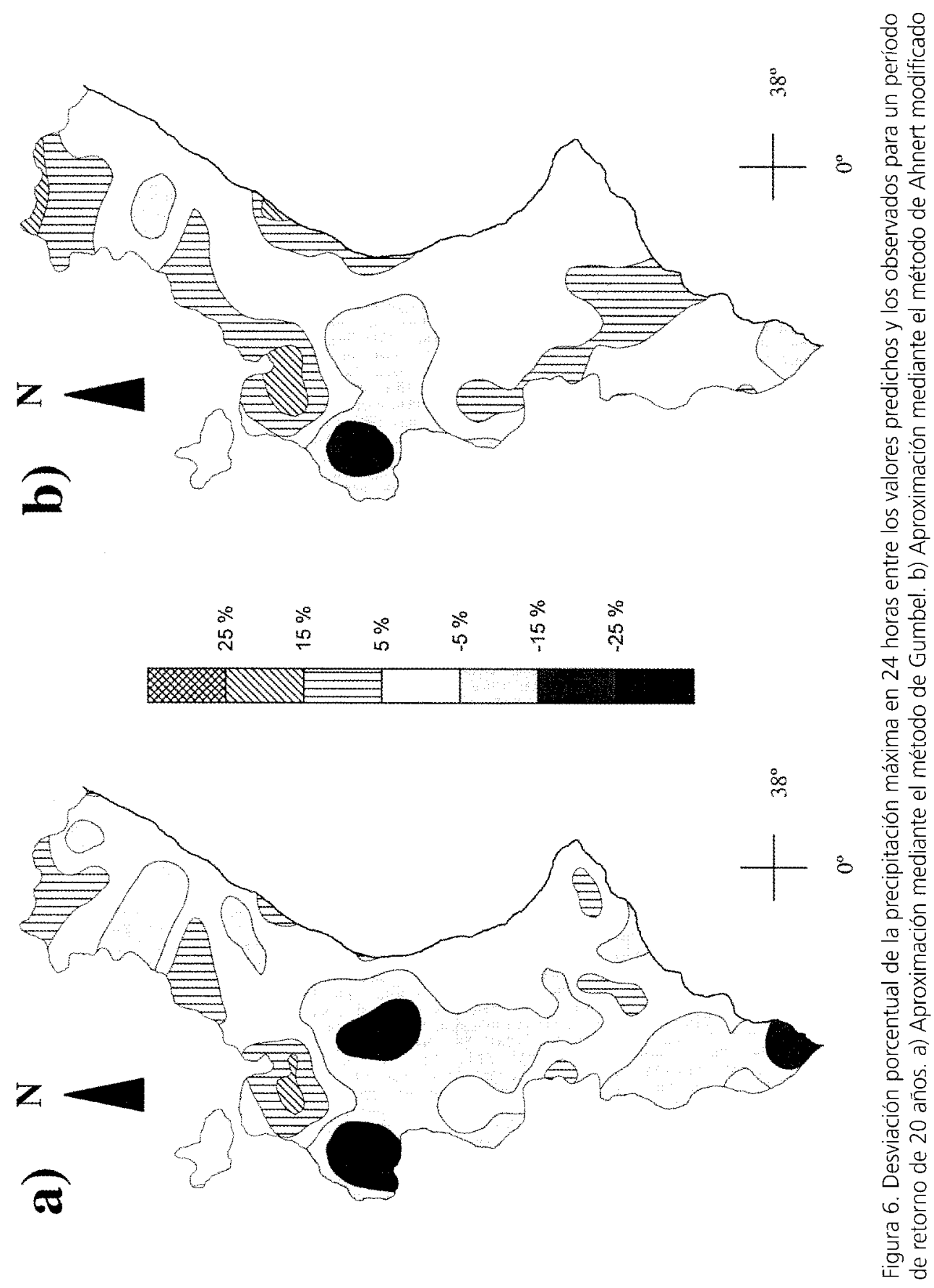



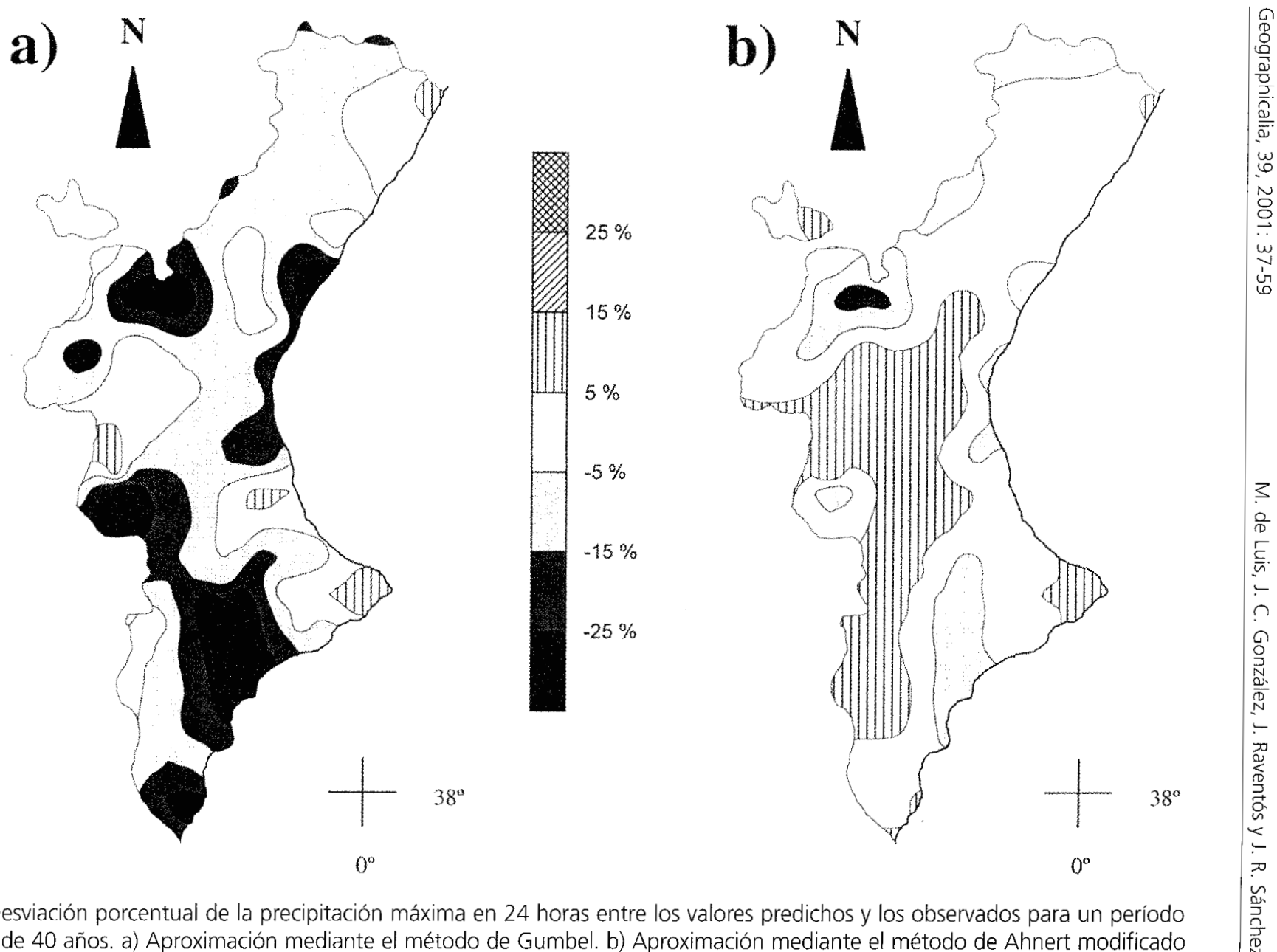

Figura 7. Desviación porcentual de la precipitación máxima en 24 horas entre los valores predichos y los observados para un período de retorno de 40 años. a) Aproximación mediante el método de Gumbel. b) Aproximación mediante el método de Ahnert modificado 
de Gumbel son las que tienen las discrepancias más acusadas y vuelven a mostrar en gran parte del territorio valenciano graves subestimaciones de los valores observados que llegan a ser en algunos sectores superiores a $-25 \%$.

Por su parte, los ajustes obtenidos mediante la propuesta modificada de Ahnert parecen ofrecer una mejor distribución de los residuos en este intervalo de retorno. Su distribución espacial es también más homogénea y las discrepancias no superan en general valores de $\pm 5 \%$.

En la Figura 8 se muestra la precipitación máxima esperada en 24 horas para un periodo de retorno de 100 años. El mapa permite observar que prácticamente en cual-

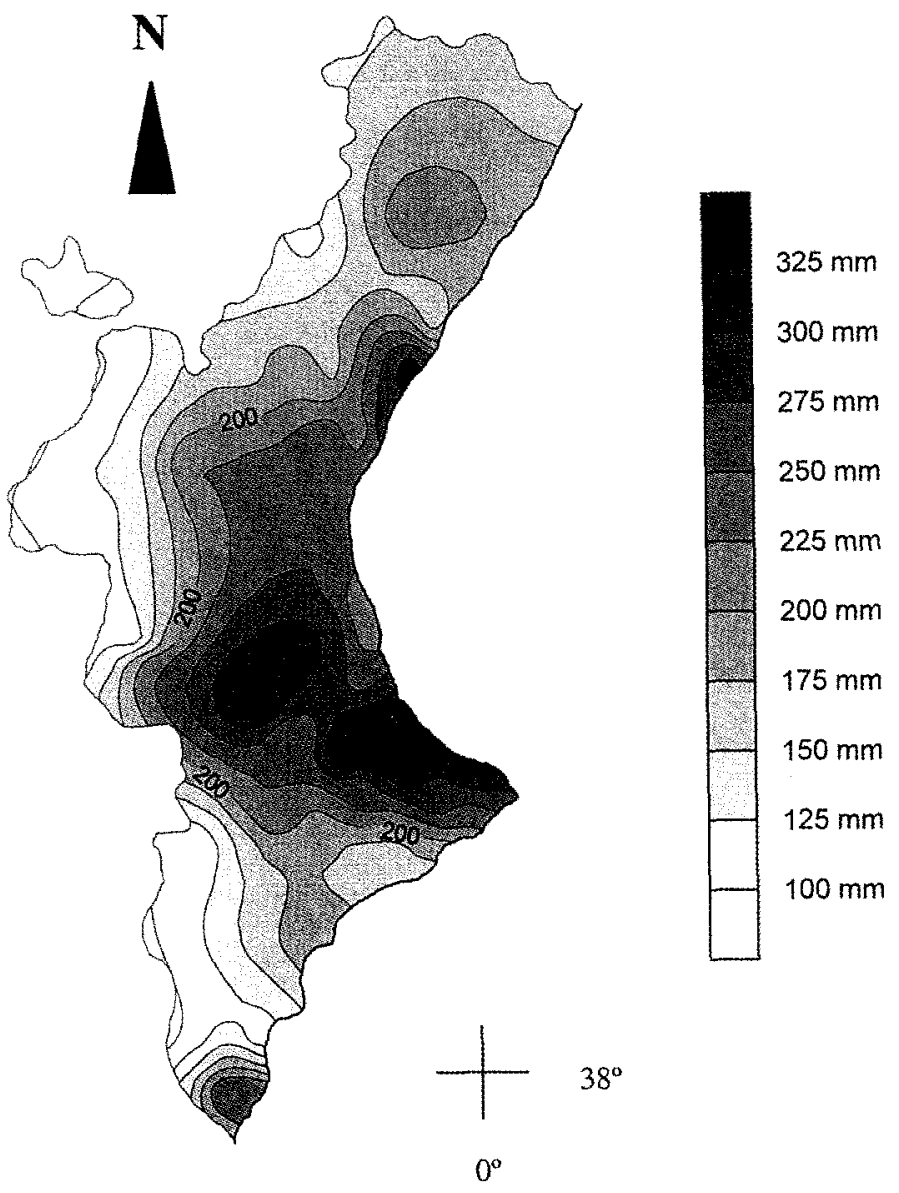

Figura 8. Precipitación máxima esperada en 24 horas en un período de retorno de 100 años 
quier punto de la Comunidad se podría producir una precipitación en 24 horas de $200 \mathrm{~mm}$, y que esta precipitación llegaría a doblarse (>400 mm) en numerosos sectores de la provincia de Valencia.

Desde la perspectiva contraria, en la figura 9 se presenta una cartografía de isócronas de los eventos diarios superiores a los $200 \mathrm{~mm}$. En el citado mapa se observa que en los sectores costeros de la provincia de Valencia tal precipitación se espera con una recurrencia inferior a los 25 años, y, en segundo lugar, que la isocrona de 100 años de este evento de precipitación incluye la práctica totalidad del territorio de la Comunidad Valenciana.

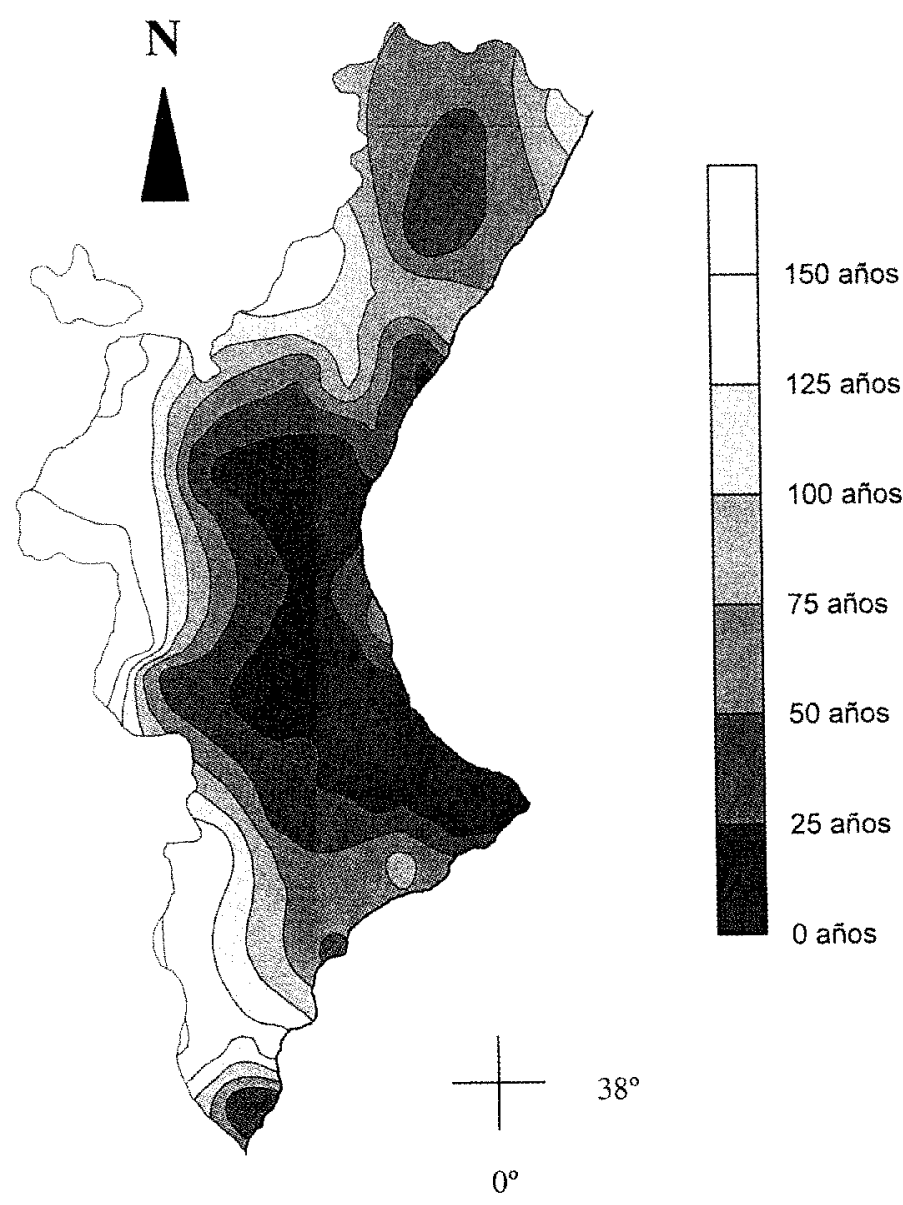

Figura 9. Período de retorno (años) de un evento de lluvia (24 horas) superior o igual a $200 \mathrm{~mm}$. Método de Anhert modificado 


\section{Discusión}

En el contexto del "Cambio Global", el estudio de los eventos extremos es uno de los aspectos más debatidos tanto en sus características (Gajic-Capka, 1991; Miroslava, 1991; Capka y Gajic-Capka, 1992, Nobilis et al., 1991; Svensson y Berndtsson, 1996; Loukas y Quick, 1996; Kieffer y Bois, 1997; White et al., 1997; Bradley, 1998; Pagliara et al., 1998, McGuffie et al., 1999; Abbs, 1999), como la evaluación de sus consecuencias (Gallart, 1995; Wainwright, 1996; Mulligan, 1998; Pagliara et al., 1998; Puigdefábregas y Mendizábal, 1998; Nyberg y Rapp, 1998; De Luís et al., 2000).

Sin embargo, en el análisis de la distribución de los sucesos extremos en el área mediterránea, y especialmente en el litoral español, debe considerarse por un lado el gran efecto que tienen los factores topográficos locales en la distribución de las precipitaciones y la elevada frecuencia de procesos convectivos generadores de fuertes aguaceros sobre los que la oposición del relieve acentúa el mecanismo de disparo (Camarasa, 1993; Martín-Vide, 1994). La Comunidad Valenciana se encuentra en uno de los sectores con mayor frecuencia de ciclogénesis del mundo (Petterssen, 1956), y la dinámica de las células convectivas puede resultar determinante en el origen y tipo del régimen pluviométrico local, pues son extremadamente móviles en el espacio, muy dinámicas en su desarrollo interno (Sharon, 1972; Yair y Lavee, 1985) y se manifiestan sobre extensiones "pequeñas" que oscilan entre los 10 y los $50 \mathrm{~km} 2$ (Camarasa, 1993). El resultado de esta situación es la extrema variabilidad espacial y temporal de las precipitaciones y en consecuencia de los eventos extremos.

En el mediterráneo español la escasez de registros impide una caracterización detallada en intervalos horarios o de minutos, pues el despliegue de los sistemas automatizados (SAIH) es muy reciente; por esta razón la escala temporal de trabajo empleada ha sido y es la diaria, tal como se manifiesta en los trabajos de Romero et al. (1998), Vilar et al., (1988) y Lana et al., (1995) en Cataluña, Erena y Rincón (1997) en la Región de Murcia, o de Martín-Vide, (1994), Gil-Olcina, (1986) y el presente trabajo, en la Comunidad Valenciana.

A tenor de los resultados obtenidos en el presente estudio, los valores máximos esperados para un periodo de retorno de 100 años superan los $200 \mathrm{~mm}$ en prácticamente todo el territorio, y rebasan los 300 ó $400 \mathrm{~mm}$ en algunos sectores. Pese a lo elevado de estas predicciones, existen ejemplos recientes que demuestran esta posibilidad, como los $160 \mathrm{~mm} / 24 \mathrm{~h}$, caídos en la ciudad de Alicante durante el 30 de septiembre de 1997, los más de $500 \mathrm{~mm}$ recogidos en algunos observatorios de la comarca de la Safor el mismo día, o los más de 800 registrados el 3 de Noviembre de 1987 en la citada comarca (Armengot-Serrano, 1994). Tales resultados y predicciones concuerdan con el hecho conocido de que en la Comunidad Valenciana no sólo se registran los máximos del conjunto peninsular (Olcina, 1994; Romero et al., 1998), sino que sus valores se aproximan a los máximos mundiales (Armengot-Serrano, 1994). 
Pese a la existencia de una variada gama de métodos avalados en la bibliografía especializada para caracterizar la magnitud-frecuencia de los eventos extremos, su aplicación a las peculiares condiciones de nuestro clima se debe realizar con la máxima de las prudencias. Normalmente, las anomalías detectadas en el ajuste de Gumbel se suelen atribuir al hecho de haber "capturado" durante el período de registro uno o más eventos de retornos centenarios. El presente trabajo sugiere, que, en el caso concreto del régimen de precipitaciones de la Comunidad Valenciana, la clásica aproximación de Gumbel pudiera no ser la más correcta, pues genera importantes subestimaciones en sus previsiones que, además, no se distribuyen normalmente. Resultados similares fueron encontrados por Lana et al. (1995) en algunos observatorios de Cataluña, quienes modificaron la aproximación estadística utilizando una distribución de Gumbel Tipo III (aún más compleja en su aplicación y en cierto modo semejante a la aquí propuesta).

En este sentido, la metodología propuesta por Ahnert en 1986 y modificada en el presente trabajo parece mostrarse como una buena alternativa. Su sencillez matemática y la posibilidad de trabajar con series parciales seleccionadas en función de cuál sea el objetivo del estudio pueden convertirla en una herramienta de gran utilidad. En nuestro caso particular, al menos, sus resultados se manifestaron óptimos en las diferentes comarcas y sectores climáticos y en los diferentes periodos de retorno considerados. Dada la gran diversidad de condiciones pluviométricas que pueden encontrarse en nuestro territorio, esta homogeneidad en las predicciones parecen conferir a este método una posible aplicación a otras regiones de regímenes semejantes.

\section{Conclusiones}

La enorme irregularidad de las precipitaciones diarias en la Comunidad Valenciana plantea dificultades en el análisis de los retornos por la persistente presencia de sesgos en los residuos de las funciones de ajuste tradicionalmente empleadas (Gumbel). El ensayo de una nueva función empírica que modifica la propuesta por Ahnert sugiere que, al menos para el dominio de la función definida por la duración de los registros históricos, se mejora notablemente la distribución de tales residuos, en vez de considerar tales sucesos como sucesos con retornos superiores a la longitud de registros empíricos. Las implicaciones que el análisis espacial de los valores obtenidos por la función corregida proporciona, indican que prácticamente el conjunto de la Comunidad debería esperar una precipitación diaria de $100 \mathrm{~mm}$ en 24 horas en períodos de 25 años. En conclusión, y pese a la prudencia que debe presidir en todos los casos cualquier intento de predicción futura basada en registros históricos, la metodología de Ahnert modificada en este estudio parece recoger de una manera más homogénea y efectiva lo peculiar del régimen pluviométrico mediterráneo de la Comunidad Valenciana. 


\section{Agradecimientos}

Los autores expresan su agradecimiento al Dr. Pérez Cueva (Dpto. Geografía, Universidad de Valencia) así como al Gobierno Regional de la Comunidad Valencia (Conselleria d'Ordenació del Territori, Generalitat Valenciana) por las facilidades dadas para acceder a las bases de datos empleadas en el presente estudio. Trabajo financiado por CICYT, Proyectos CLI95-1947-CO3-O3 y CLI99-0957, y Generalitat Valenciana, Projecte GV97-RN-14-2. Los autores agradecen asimismo la ayuda otorgada por el Instituto de Cultura Juan Gil-Albert (Alicante).

\section{Bibliografía}

Abbs, D.J. (1999): A numerical modeling study to investigate the assumptions used in the calculation of probable maximum precipitation. Water Resources Research, 35, p. 785-796.

Al-Mashidani, G., Pande, B.B.L., and Mujda, M.F. (1978): A Simple Version of Gumbel 's Method for Flood Estimation. Hydrological Sciences Bulletin, 23, p. 373380.

Arnbjergnielsen, K., Harremoes, P., and Spliid, H. (1994): Non-Parametric Statistics on Extreme Rainfall. Nordic Hydrology, 25 , p. $267-278$.

Ahnert, F. (1986): An aproach to the identification of morphoclimates. En: Gardiner, V., (ed.): International Geomorpbology, John Wiley \& Son, p. 159-188.

Armengot-Serrano, R. (1994): Las precipitaciones extraordinarias. En Pérez Cueva, A. (ed): Atlas climàtic de la Comunitat Valenciana. Conselleria d'Obres Públiques, Urbanisme i Transports. Generalitat Valenciana, Col.leció Territori, n. ${ }^{\circ} 4$.

Benjamin, J.R. (1970): Probability, Statistics and Decision for Civil Engineers. McGraw-Hill, New York.

Bradley, A. A. (1998): Regional frequency analysis methods for evaluating changes in hydrologic extremes. Water Resources Research, 34, p. 741-750.

Camarasa, A. (1993): La estructura interna de las tormentas Mediterráneas. Cuadernos de Geografia, 54, p. 169-188.

Capka, B. and Gajic-Capka, M. (1992): A case analysis of heavy rainfall causing flash floods. Theoretical and Applied Climatology, 45, p. $77-82$.

Chow, V.T. (1951): A general formula for hydrologyc frequency analysis. Transactions of the American Geopbysical Union, 32, p. 231.

Chow, V.T. (1956): Handbook of Applied Hydrology. New York, McGraw-Hill Book Co.

Chu, P.S. and Wang, J.X. (1998): Modeling return periods of tropical cyclone intensities in the vicinity of Hawaii. Journal of Applied Meteorology, 37, p. 951-960.

De Luís, M. (2000): Estudio espacial y temporal de las tendencias de lluvia en la Comunidad Valenciana (1961-1990). Logroño, Geoforma.

De Luís, M., Raventós, J., González-Hidalgo, J.C., Sánchez, J.R., Cortina, J., and GarcíaCano, M.F. (1998): Analysis of maximum daily probability precipitation for different return periods: Comparison among three 
different methods on Mediterranean conditions. Proc. 15th International Sedimentological Congress. Publicaciones de la Universidad de Alicante, p. 287-288.

De Luís, M., García-Cano, M.F., Cortina, J., González-Hidalgo, J.C., Sánchez, J.R. and Raventós, J. (2000): Climatic trends, disturbances and succesion in a mediterranean shrubland. Forest Ecology and Management, (Número monográfico, p. $1-18$, en prensa).

De Ploey, J., Kirkby, M.J. and Ahnert, F. (1991): Hillslope erosion by rainstorms: a magnitude-frequency analysis. Earth Surface Processes and Landforms, 16, p. 399-409.

Dupuis, D.J. (1997): Extreme value theory based on the $r$ largest annual events: a robust approach. Journal of Hydrology, 200, p. 295-306.

Erena, M. and Rincón, L. (1998): Spatial and temporal distribution of the rainfall in the Murcia region. En Conference on Global Change The Earth's changing land. GCTE-LUCC, Open Science. Barcelona, Spain.

Escalante-Sandoval, C. (1998): Multivariate extreme value distribution with mixed Gumbel marginals. Journal of the American Water Resources Association, 34, p. 321-333.

Esteban-Parra, M.J., Rodrigo, F.S., and CastroDiez, Y. (1998): Spatial and temporal patterns of precipitation in Spain for the period 1880-1992. International Journal of Climatology, 18, p. 1557-1574.

Gajic-Capka, M. (1991): Short-term precipitation maxima in different precipitation climate zones of Croatia, Yugoslavia. International Journal of Climatology, 11, p. 677-687.

Gallart, F. (1995): The relative geomorphic work affected by four processes in rainstorms: A conceptual approach to magni- tude and frequency. Catena, 25, p. 353364.

Gil-Olcina, A. (1986): Previsión de precipitaciones máximas según la ley de Gumbel. En Gil Olcina, A (ed): Inundaciones en la Ciudad y Término de Alicante. Alicante, Instituto Universitario de Geografía y Ayuntamiento de Alicante, p. 95-101.

Goodess, C.M. and Palutikof, J.P. (1998): Development of daily rainfall scenarios for southeast Spain using a circulationtype approach to downscaling. International Journal of Climatology, 18, p. 1.051-1.083.

Goyal, S.C. and Kathuria, S.N. (1984): Return Period Analysis of Extreme Rainfall Events. Mausam, 35, p. 531-536.

Gumbel, E.J. (1958): Statistis of Extremes. Columbia University Press.

Hershfield, D.M. (1961): Estimating the probable maximum precipitation. Journal Hydraulic. Division American Civil Engineering, 2933, p. 99-116.

Hershfield, D.M. (1965): Method for estimating probable maximum rainfall. Journal Water Works Association, 57, p. 105-112.

Hershfield, D.M. (1979): Secular Trend in Extreme Rainfalls. Journal of Applied Meteorology, 18, p. 1.078-1.081.

Houghton, J.T., Meira Filho, L.G., Callander, B.A., Harris, N., Kattenberg, A. and Maskell, K. (eds.). (1996): Climate change 1995: the sciencie of climate change. Cambridge University Press, UK.

Iwashima, T. and Yamamoto, R. (1993): A statistical analysis of the extreme events: long term trend of heavy daily precipitation. Journal of the Meteorological Society of Japan, 71, p. 637-640.

Karl, T.R., Knight, R.W., and Plummer, N. (1995): Trends in high-frequency climate variability in the twentieth century. Nature, 377, p. 217-220. 
Kieffer, A. and Bois, P. (1997): Variability of the statistical characteristics of extreme rainfall values in the French Alps. Rev. Science Eau, 10, p. 199-216.

Laita, M. and Grimalt, M. (1997): Vorticity and preassure anomalies in the western Mediterranean during El Niño/Southern Oscillation extremes. International Journal of Climatology, 17, p. 475-482.

Lana, X., Fernández-Mills, G., and Burgueno, A. (1995): Daily precipitation maxima in Catalonia (north-east Spain): Expected values and their spatial distribution. International Journal of Climatology, 15 , p. 341-354.

Lewis, R.-P.W. (1992): Flooding at Carmarthen in October 1987: Historical Precedents and Statistical Methods. Weatber, 47, p. 82-89.

Llamas, J., Charbonneau, R., and Rassam, J.C. (1987): Statistical Analysis of Extreme Events (Analyse Statistique d'Evenements Extremes), En: Water for the Future: Iydrology in Perspective. Washington, International Association of Hydrological Sciences, p. 71-91.

Llasat, M.C. and Puigcerver, M. (1997): Total rainfall and convective rainfall in Catalonia, Spain. International Journal of Climatology, 17, p. 1683-1695.

Loukas, A. and Quick, M.C. (1996): Spatial and temporal distribution of storm precipitation in southwestern British Columbia. Journal of Hydrology, 174, p. 37-56.

Madsen, H., Mikkelsen, P.S., Rosbjerg, D., and Harremoes, P. (1998): Estimation of regional intensity-duration-frequency curves for extreme precipitation. Water Science and Tecbnology, 37, p. 29-36.

Martín-Vide, J. (1994): Precipitaciones: máximos diarios probables e irregularidad. En: Atlas climatic de la Comunitat Valenciana. Conselleria d'Obres Públiques, Urbanisme i Transports.
Generalitat Valenciana, Col.leció Territorio, No 4, p. 94-96.

Martín-Vide, J. and Gómez, L. (1999): Regionalization of peninsular Spain based on the length of dry spells. International Journal of Climatology, 19, p. 537-555.

McGuffie, K., Henderson-Sellers, A., Holbrook, N., Kothavala, Z., Balachova, O., and Hoekstra, J. (1999): Assessing simulations of daily temperature and precipitation variability with global climate models for present and enhanced greenhouse climates. International Joumal of Climatology, 19, p. 1-26.

Miroslava, U. (1991): The extreme value distribution of 5-min. rainfall data at Belgrade. Theoretical and Applied Climatology, 44, p. 223-228.

Mitchell, J.F.B. (1989): The greenhouse effect and climate change. Review of Geopbysics, 27, p. 115-139.

Mulligan, M. (1998): Modelling the geomorphological impact of climatic variability and extreme events in a semi-arid environment. Geomorpbology, 24, p. 59-78.

Nobilis, F., Haiden, T. and Kerschbaum, M. (1991): Statistical considerations concerning probable maximum precipitation (PMP): in the alpine country of Austria. Theoretical and Applied Climatology, 44, p. 89-94.

Nyberg, R. and Rapp, A. (1998): Extreme erosional events and natural hazards in Scandinavian mountains. Ambio, 27, p. 292-299.

Olcina, J. (1994): Riesgos Climáticos en la Península Ibérica. Madrid, Libros Penthalon.

Pagliara, S., Viti, C., Gozzini, B., Meneguzzo, F., and Crisci, A. (1998): Uncertainties and trends in extreme rainfall series in Tuscany, Italy: Effects on urban drainage networks design. Water Science and Technology, 37, p. 195-202. 
Pérez-Cueva, A. (Dir): (1994): Atlas climàtic de la Comunitat Valenciana. Conselleria d'Obres Públiques, Urbanisme i Transports. Generalitat Valenciana, Colleció Territorio, n. ${ }^{4}$, p. 98-99.

Petterssen, S. (1956): Weather Analysis and Forecasting, Vol. I. Nueva York, McGrawHill Company.

Puigdefábregas, J. and Mendizabal, T. (1998): Perspectives on desertification: Western Mediterranean. Journal of Arid Environments, 39, p. 209-224.

Quereda, J. (1994): ¿Hacia un cambio climático? Castellón, Publicaciones de la Universitat Jaume I.

Reich, B.M. (1970): Flood series compared to rainfall extremes. Water Resources Research, 6, p. 1655-1667.

Reich, B.M. (1973): How frequently will floods occur. Water Resources Bulletin, 9, p. 187-188.

Rodriguez-Puebla, C., Encinas, A.H., Nieto, S., and Garmendia, J. (1998): Spatial and temporal patterns of annual precipitation variability over the Iberian Peninsula. International Journal of Climatology, 18, p. 299-316.

Romero, R., Guijarro, J.A., Ramis, C., and Alonso, S. (1998): A 30-year (1964-1993) daily rainfall data base for the Spanish Mediterranean regions: First exploratory study. International Journal of Climatology, 18, p. 541-560.

Rowntree, P.R. (1988): Review of general circulation models as a basin for predicting the effects of vegetation change on climate. En: Reynolds, E.R.C. \& Thompson, F.B. (eds.), Forest, Climate and Hydrology: Regional Impacts. Singapur, Kefford Press, p. $162-203$

Rowntree, P.R., Murphy, J.M. and Mitchell, J.F.B. (1993): Climate change and future rainfall predictions. Journal. Institue
Water Environmental Management, 7, p. 464-470.

Sevruk,B. y Geiger, H. (1981): Selection of distribution types for extremes of precipitation, Ginebra, WMO, Operational Hydrology, Report, n. 15.

Sharon, D. (1972): The spottiness of rainfall in a desert area. Joumal of Hydrology, 17, p. 161-175.

Slimani, M. and Lebel, T. (1987): Comparison of Three Methods of Estimating Rainfall Frequency Parameters According to the Duration of Accumulation. Regional Flood Frequency Analysis: Proceedings of the International Symposium on Flood Frequency and Risk Analyses. Boston, MA., Louisiana State University, Baton Rouge, p. 277-291.

Suppiah, R. and Hennessy, K.J. (1998): Trends in total rainfall, heavy rain events and number of dry days in Australia, 19101990. International Journal of Climatology, 18, p. 1141-1164.

Svensson, C. and Berndtsson, R. (1996): Characterization of extreme rainfall in an East Asian monsoon-climate catchment in the upper reaches of the Huai River, China. International Journal of Climatology, 16, p. 321-337.

Vilar, E., Burgueño, A., Puigcerver, M. and Austin, J. (1988): Analysis of join rainfall rate duration statistics: microwave system design implications. J.E.E.E. Trans. Comm., 36 (6), p. 650-661.

Wainwright, J. (1996): Infiltration, runoff and erosion characteristics of agricultural land in extreme storm events, SE France. Catena, 26, p. 27-47.

White, S., García-Ruiz, J.M., Marti, C., Valero, B., Errea, M.P., and Gómez-Villar, A. (1997): The 1996 Biescas campsite disaster in the central Spanish Pyrenees, and its temporal and spatial context. Hydrological Processes, 11, p. 1.797-1.812. 
Wigley, T.M. (1985): Impact of extreme events. Nature, 316, 106-107.

W.M.O. (1986): Manual for estimation of probable maximum precipitation. W.M.O., Operational Hydrology, Report, Ginebra, n. ${ }^{\circ} 1$ (segunda edición).

Yair, A. and Lavee, H. (1985): Runoff generation in arid and semi-arid zones. In Anderson and Burt (Eds.): Hydrological Forecasting, p.183-220.
Yu, P.S. and Cheng, C.J. (1998): Incorporating uncertainty analysis into a regional IDF formula. Hydrological Processes, 12, p. 713-726.

Yu, B. and Neil, D.T. (1993): Long-Term Variations in Regional Rainfall in the South-West of Western Australia and the Difference Between Average and High Intensity Rainfalls. International Journal of Climatology, 13. 